\title{
Una gestión activa de los intangibles empresariales y su incidencia en los resultados financieros de un modo sostenible
}

\author{
Lidia García-Zambrano a ${ }^{\text {a }}$ Arturo Rodríguez-Castellanos ${ }^{a}$, José \\ DOMINGO GARCÍA-MERINO ${ }^{\text {a }}$ \\ a Universidad del País Vasco, Facultad de CC.EE., C/ Lehendakari Aguirre, 8348015 Bilbao, \\ España.E-mail: lidia.garcia@ehu.es, arturo.rodriguez@ehu.es, josedomingo.garcia@ehu.es
}

\begin{abstract}
RESUMEN
En la actualidad, es comúnmente aceptado que la dotación de recursos y capacidades de carácter intangible se ha convertido en el elemento diferenciador entre empresas. Sin embargo, sólo un grupo reducido permiten la obtención de una ventaja competitiva siendo son la fuente principal de creación de valor, las denominadas competencias básicas. La mayor parte de los estudios que relacionan los intangibles con los resultados de las empresas consideran únicamente el stock que posee la empresa, y no analiza la gestión llevada a cabo. Nuestro objetivo es verificar si una gestión más eficiente de estos recursos tiene una incidencia en los resultados financieros, medidos a través del ROA, de un modo sostenible. Para alcanzar este objetivo, se llevó a cabo un estudio de campo, realizando llamadas telefónicas a los directivos financieros de las empresas del País Vasco, obteniéndose evidencia positiva a favor de la hipótesis planteada.

Palabras clave: Intangibles, competencias básicas, gestión del capital intelectual, resultados financieros.
\end{abstract}

\section{Management of Firms' Intangibles and Their Impact on Sustainable Financial Performance}

\begin{abstract}
It is widely accepted that the allocation of intangible resources and capabilities has become the difference between firms. However, only a few of them allow to gain a competitive advantage, so-called core competencies. Most of the studies about to the intangibles and firms performance consider only the stock held by the company, and they do not considered intangibles management. Therefore, our objective is to test whether the core competencies management translates into improved sustainably performance, measured through ROA. To this end, a field study was performed, by making telephone calls to Basque Country companies' financial managers, and favorable evidence was obtained.

Keywords: Intangible, Core Competencies, Intellectual Capital Management, Financial Performance.

Clasificación JEL: M21, G39

\footnotetext{
* Esta investigación fue llevada a cabo por el Grupo de Investigación de la Valoración Económico-Financiera de los intangibles (VALINTE) de la Universidad del Pais Vasco. Agradecemos a los miembros del Foro Vasco de Gestión y Finanzas por su colaboración en el tratamiento del cuestionario utilizado para obtener información de los directivos financieros sobre algunos temas analizados en este trabajo.
}

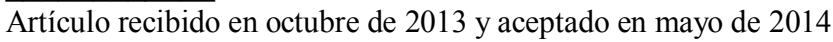

Artículo disponible en versión electrónica en la página www.revista-eea.net, ref. ə-32303 


\section{INTRODUCCIÓN}

En la actualidad, la dotación de recursos materiales y financieros de las empresas no constituye un elemento diferenciador y no permite la obtención de ventajas competitivas sostenibles, siendo los intangibles la fuente de competitividad básica empresarial (Lev y Zambon 2003; Corrado et al., 2009), especialmente aquellos intangibles basados en el conocimiento.

Las empresas han respondido a este entorno competitivo en dos sentidos: deshaciéndose de las estructuras integradas y dando mayor peso e importancia a la gestión de los flujos de conocimiento. La integración vertical de las empresas de la era industrial se ha sustituido paulatinamente por una red de estrechas colaboraciones. Las tradicionales economías de escala deben ser complementadas, y en ocasiones, reemplazadas por unas amplias economías de red. La importancia del conocimiento y de los recursos de carácter intangible debe tener su reflejo y ponerse de manifiesto de un modo diferenciador entre la gestión de las empresas que forman parte de la "vieja" y la "nueva" economía (Bose y Thomas, 2007). Las empresas deben enfocar su gestión hacia los intangibles y la generación de conocimiento organizativo, como base para fortalecer su competitividad (Tejedo y Alfaro, 2014). El éxito y el liderazgo, inclusive en sectores tradicionales, se sustentan en la gestión del conocimiento de la cadena de valor en la cual la empresa participa (Prahalad, 1983; Holsapple y Singh, 2001, Cepeda, 2006; Holsapple et al., 2007).

Existen numerosos trabajos que recogen la existencia de una relación entre el stock de intangibles y los resultados empresariales (Jardon y Martos, 2008). De hecho, tal y como muestran trabajos como García-Merino et al. (2008) y Steenkamp y Kashyap (2010), el interés por los intangibles no se produce únicamente en la comunidad académica, sino que también hay un creciente interés por parte de la comunidad empresarial.

Sin embargo, para entender la capacidad de las empresas de lograr una ventaja competitiva de un modo sostenible, más allá del stock de conocimiento resulta relevante el análisis del flujo de conocimiento (Rastogi, 2000). Es necesario llevar a cabo un análisis dinámico de flujos de generación de conocimiento frente al planteamiento estático tradicional orientado al stock de conocimiento existente (Seleim y Khalil, 2011). El mantenimiento de una posición competitiva está directamente vinculado a la gestión del stock de conocimientos controlado por la empresa. La capacidad de adquirir y aplicar el saber y los conocimientos, constituyen la nueva fuente de riqueza (Handy, 1994). La importancia de la gestión de los intangibles, desde un visto estratégico, y su vinculación con los resultados empresariales prácticamente no ha sido abordada en la literatura (Kianto et al., 2013), siendo éste el planteamiento que se llevará cabo en este trabajo. 
Por otro lado se ha de tener en cuenta que no todos los intangibles tienen igual importancia; siendo las denominadas competencias básicas la base de la ventaja competitiva de las empresas, y por tanto sobre las que la empresa debe centrar su gestión. Cabe esperar que una gestión adecuada de estas competencias básicas permita a las empresas obtener ventajas competitivas y mejores resultados económicos, de un modo sostenible. El objetivo de nuestra investigación es establecer una relación entre la gestión de los intangibles, en concreto aquellos que constituyen una competencia básica, y el resultado económico.

Para cumplir este objetivo, llevamos a cabo una encuesta a directivos financieros del País Vasco, para recoger información sobre los esfuerzos que han llevado a cabo en la gestión y fortalecimiento de sus competencias básicas, y se ha analizado los resultados financieros logrados por estas empresas. Los resultados del análisis confirman que las empresas que gestionan de un modo más activo sus intangibles obtienen un mejor resultado.

Este trabajo se estructura como sigue: en la siguiente sección, tras con una breve explicación del enfoque de Recursos y Capacidades, se justifica el papel cada vez más relevante que tienen los intangibles en la competitividad empresarial, y sobre los resultados empresariales. A continuación se detalla la metodología seguida en este estudio, describiéndose la muestra empleada y el procedimiento empleado. En la cuarta sección se analizan los resultados obtenidos, $\mathrm{y}$, finalmente, se presentan las concusiones del estudio.

\section{LOS RECURSOS INTANGIBLES BASADOS EN LA ECONOMÍA DEL CONOCIMIENTO}

\subsection{El impacto decisivo de los intangibles en la competitividad empresarial: un enfoque basado en recursos}

En dirección estratégica coexisten dos perspectivas complementarias para entender la creación de valor: el enfoque de la Organización Industrial (Porter, 1980, 1985) y el enfoque basado en los Recursos (Wernelfelt, 1984; Barney, 1986; Prahalad y Hamel, 1990). Durante las dos últimas décadas, se ha producido un cambio fundamental en el campo de la dirección estratégica a la hora de explicar las diferencias en los resultados inter-empresariales, pasando de un planteamiento centrado en el sector a un planteamiento basado en los recursos (Hoopes et al., 2003). Siguiendo este enfoque, la dotación de recursos, el seguido mayoritariamente para explicar la obtención de rendimientos anormales.

Sin embargo, no todos los recursos presentan la misma importancia para el éxito empresarial. Solo los recursos con ciertas características pueden generar una posición diferenciadora respecto de los competidores. Los recursos que presenten valor, escasez, inimitabilidad, y no-sustituibilidad (requisitos VRIN) son considerados "recursos críticos" (Wernefelt, 1984), "factores estratégicos" 
(Barney, 1986), activos estratégicos (Amit y Schoemaker, 1993), "recursos estratégicos" (Peteraf, 1993). Los intangibles son aquellos que, debido a su falta de base física o financiera y que requieren un proceso de generación y acumulación lento, que más fácilmente cumplen los requisitos del criterio VRIN, y por tanto más frecuentemente se convierten en factores básicos de competitividad (Lev, 2001).

Blair y Wallman (2001, p. 3) definen los recursos intangibles como "factores no físicos que contribuyen o son empleados en la producción de bienes y prestación de servicios, o se espera de ellos que generen beneficios futuros para los individuos o empresas que controlan el uso de estos factores".

Así como una derivación del RBV, se desarrolla el enfoque basado en los recursos Intangibles o también denominado enfoque basado en el Capital Intelectual (Hudson, 1993; Brooking, 1996; Bontis, 1996, 1998; Edvinsson y Malone, 1997; Stewart, 1997; Roos et al., 1997; Reed et al., 2006; Martín et al., 2011). El enfoque basado en los recursos Intangibles (Reed et al., 2006) toma como base la RBV y entiende que los intangibles constituyen el recurso clave para el logro de resultados empresariales superiores sostenibles en el tiempo.

Se ha de tener en cuenta que los recursos no son productivos por sí mismos. $\mathrm{Su}$ valor se obtiene en conjunción con otros recursos, dentro de un grupo de trabajo y con un objetivo concreto. Los recursos pueden ser considerados como inputs del proceso productivo (Grant, 1991). Es la combinación de recursos, en un contexto competitivo específico, lo que determina las capacidades de una empresa. La creación de valor añadido y el logro de una ventaja competitiva, no sólo depende de la base de recursos que posea la empresa, sino de su habilidad para movilizar, coordinar, combinar e integrar esos recursos dentro de la organización (Sirmon et al., 2008). De ello se deduce que el verdadero elemento diferenciador lo constituyen las capacidades que poseen las empresas. Las capacidades organizativas por definición están asociadas al conocimiento que posee la empresa, y son de carácter intangible.

Las capacidades asociadas intangibles constituyen lo que se denomina capital intelectual. Sánchez-Medina (2003) define el capital intelectual como combinación de recursos inmateriales o intangibles de una organización, incluyendo el conocimiento personal, la capacidad para aprender y adaptarse, las relaciones con los clientes y los proveedores, las marcas, los procesos internos, la capacidad de I+D, etc., que, reflejados o no en los estados financieros, están directa o indirectamente controlados por dicha organización y generan o generarán valor futuro para la misma, de forma que sobre ellos se podrá sustentar una ventaja competitiva sostenida. Aunque los recursos intangibles basados en el conocimiento o capital intelectual pueden clasificarse y agruparse siguiendo diferentes criterios, el más habitual diferencia entre Capital Humano, Capital Estructural y Capital Relacional. 
Dentro del conjunto de capacidades de una empresa, Prahalad y Hamel (1990) diferencian entre aquellas capacidades que inciden de manera importante en los resultados de la empresa, lo que ellos denominan competencias nucleares o competencias básicas (core competencies), y aquéllas que tienen un carácter periférico. Las competencias básicas son el conjunto de habilidades o aptitudes desarrolladas por la empresa que generan un significativo valor o beneficio para el cliente (Prahalad y Hamel, 1990, 1994).

Por tanto, entendemos que las competencias básicas asociadas al capital intelectual constituyen la fuente de conocimientos o actividades que, al proporcionar ventaja competitiva, son determinantes en la creación de valor, y por tanto sobre las que las empresas deberán centrar sus esfuerzos.

\subsection{Relación entre las competencias básicas intangibles y el valor empresarial}

Tal y como se ha señalado, la generación y el mantenimiento de ventajas competitivas, las cuales determinan la capacidad para la creación de valor para los propietarios de la empresa, están fuertemente interrelacionadas con la realización de inversiones en intangibles (García-Parra, et al., 2004).

Son numerosos los estudios que analizan la relación entre el capital intelectual y los resultados (Bontis et al., 2000; Firer y Williams, 2003; Wang y Chang, 2005; Cohen y Kaimenakis, 2007; Cabrita y Bontis, 2008; Jardon y Martos, 2008; Sharabati et al., 2010; Maditinos et al., 2011; Mention y Bontis, 2013), obteniendo resultados dispares.

Así por ejemplo los trabajos de Firer y Williams (2003) y Sharabati, et al. (2010) encuentran soporte a esta relación. El primero de ellos, realizado en el mercado sudafricano obtiene que existe una relación entre el valor de los intangibles y distintas medidas de resultados (ROA, Ventas/activo total) a partir de 75 empresas de cuatro sectores intensivos en conocimiento. Sharabati, et al. (2010), en el sector farmacéutico, encuentran relación entre el capital intelectual, y sus distintas dimensiones y los resultados empresariales, para ello emplea un modelo de ecuaciones estructurales, sobre una muestra de 132 directivos de empresas farmacéuticas de Jordania.

Otros trabajos no obtienen resultados tan concluyentes. Así, Bontis et al. (2000) encuentra que únicamente el capital estructural incide un modo estadísticamente significativo sobre los resultados, para lo cual emplea un modelo de ecuaciones estructurales sobre una muestra de 107 empresas malayas. Por su parte, Cohen y Kaimenakis (2007) estudiaron la relación entre el capital intelectual y los resultados, en el caso de una muestra de 52 PYME griegas. Estos autores dividen las distintas tipologías de capital intelectual en tres categorías, intangibles duros, suaves y funcionales, concluyendo que solo el primero de ellos tiene un impacto positivo estadísticamente significativo en los resultados. 
Wang y Chang (2005), en el sector de las tecnologías de la información de Taiwan, encuentran que existe una relación positiva significativa entre el capital procesos y el capital relacional pero la relación no es significativa en el caso del capital humano. Resultados similares obtiene Cabrita y Bontis (2008) en el mercado portugués, con una muestra de 253 encuestas pertenecientes a 53 empresas. Jardon y Martos (2008) encuentran que únicamente existe relación entre el capital relacional y los resultados empresariales, en 117 empresas argentinas. El capital relacional está influenciado por el capital estructural, y éste a su vez con el capital humano, siendo por tanto indirecto el impacto de estas dos dimensiones sobre los resultados empresariales. Por su parte, Maditinos, et al. (2011), a partir de una muestra de 96 empresas griegas pertenecientes a cuatro sectores diferentes encuentra que el valor de los intangibles, recogido a través del VAIC, no se muestra significativo para explicar el ROA, obteniendo que únicamente el valor del capital humano, recogido a través del VACA, es estadísticamente significativo. Mention y Bontis (2013) estudian el sector bancario en Bélgica y Luxemburgo, y sólo encuentran una relación significativa entre el capital humano y los resultados financieros obtenidos, mientras que las otras dos dimensiones no tienen una incidencia estadísticamente significativa, aunque la interacción de estas dos dimensiones si es significativa.

A pesar de este acuerdo generalizado sobre la importancia de los recursos intangibles, los trabajos señalados analizan el stock de intangibles, y no la gestión de los mismos, y su incidencia en los resultados.

Aunque desde los años 90 han proliferado el desarrollo de modelos de gestión y medición de los intangibles (ver Sveiby, 2011), los cuales han sido aplicados tanto en empresas (Stewart y Mohamed, 2001; Adams y Martin, 2009; Simaro, 2012; Burton et al, 2013) como en instituciones públicas (Fazlagic, 2005; Jones et al., 2009; Ramirez et al., 2011) y en territorios (Andriessen y Stam, 2005; Lópéz y Nevado, 2008; Ståhle, y Ståhle, 2012). Sin embargo, estos trabajos no muestran evidencias sobre los beneficios que obtienen las empresas de llevar a cabo estas prácticas. Los pocos trabajos existentes que hacen referencia a los resultados obtenidos y la gestión y medición de los intangibles analizan el efecto que la revelación de información pública sobre los intangibles tiene sobre el rendimiento empresarial, obteniéndose resultados dispares. Así, Williamns (2001) y Sonnier et al (2007) no encuentran relación entre la cantidad de información divulgada sobre el capital intelectual y el rendimiento obtenido por las empresas. Por su parte, García-Meca et al. (2005), Li et al. (2008), An et al. (2011) y Majdalany y Henderson (2014) muestran la existencia de una relación positiva, significativa, entre la divulgación de información sobre los intangibles y la rentabilidad que obtienen las empresas. Otros trabajos como el de Mangena et al. (2010) recogen evidencia a favor de una reducción del coste de capital cuando las empresas revelan información voluntaria sobre sus intangibles. Ramírez et al. (2011) concluyen que, en opinión de los responsables de 
las universidades públicas españolas, la divulgación de información sobre capital intelectual conllevaría una mejora en la satisfacción de los usuarios, en la credibilidad, imagen y reputación de estas instituciones.

A pesar de estos trabajos, consideramos que la relación gestión del capital intelectual y los resultados obtenidos no ha sido convenientemente abordado. El concepto de gestión del capital intelectual a menudo se solapa con el de gestión del conocimiento (Chatzkel, 2001; Wiig, 1997a, 1997b; Koening, 2000; Kujansivu, 2008). La gestión del conocimiento se refiere a una perspectiva táctica y operativa, y está orientada a las actividades relacionadas con la gestión y facilitación de la creación y difusión del conocimiento, mientras que la gestión del capital intelectual se centra en un aspecto en un aspecto más estratégico (Wiig, 1997a). De acuerdo con la definición por Wiig (1997a), la gestión del capital intelectual se centra en construir y gestionar los activos intelectuales desde una perspectiva de dirección estratégica empresarial. Su función es cuidar de un modo de holístico el capital intelectual de la empresa. La gestión del capital intelectual incluye diferentes actividades como la identificación, la medición, el control y el desarrollo de los intangibles empresariales (Kujansivu, 2008). Dado que los intangibles constituyen la principal fuente de ventaja competitiva consideramos que su gestión debería redundar en una mejora en los resultados, tal y como sugieren Peña (2002) o Tull y Dumay (2007).

Dado el planteamiento estratégico que presenta la gestión del capital intelectual consideramos que su incidencia en los resultados puede no ser inmediata y puntual, sino que debería tener una incidencia en el rendimiento obtenido en un horizonte temporal más amplio. Como señala Sullivan (1998) el capital intelectual no solo tiene incidencia en el resultado actual sino que define su futuro. De hecho muchas de las actuaciones relacionadas con el fortalecimiento del capital intelectual pueden tener un efecto negativo en el resultado inmediato, ya que actividades como la formación del personal, o actividades de publicidad son consideradas como gasto del ejercicio, y no como inversión. Respecto a cuál debe ser el horizonte temporal a emplear, la casuística es muy variada. Así, a modo de ejemplo recoger como Riahi-Belkaoui (2003) emplea un horizonte temporal de cinco años, para medir la relación entre el capital intelectual y el ROA. Por su parte, Wang (2011) mide la relación entre el VAIC y la rentabilidad en el mercado taiwanés durante siete años. Joshi et al. (2013) emplea un horizonte temporal de tres años para medir si el stock de capital intelectual incide en los resultados.

Por otro lado, a pesar de que la mayor parte de las empresas que han empleado modelos de gestión de intangibles presentan un perfil concreto, empresas grandes, pertenecientes a sectores intensivos en conocimientos (Castilla y Rodriguez, 2014) consideramos que dado que los intangibles se han mostrado 
relevantes en distintos sectores, independientemente del tamaño de las empresas, su gestión redundará en un mejores resultados en todo tipo de empresas.

Por todo lo anterior, planteamos la siguiente hipótesis de trabajo:

$\mathrm{H}_{1}$ : Las empresas que gestión de un modo más activo sus competencias básicas obtendrán mejores resultados de un modo sostenible.

\section{METODOLOGÍA}

Para poder contrastar las hipótesis, el proceso de investigación se organizó de la siguiente forma.

En primer lugar, se realizó una encuesta telefónica realizada entre los días 20 de noviembre de 2007 y 14 de enero de 2008, donde se recogía la opinión de directivos sobre diversos aspectos relativos a la importancia de los intangibles y la gestión proactiva de las competencias básicas. La encuesta se centró en empresas del País Vasco, en concreto en aquellas empresas con más de 10 empleados y cuyo volumen de facturación superase los 2 millones de euros anuales. Las empresas que no cumplían estas condiciones fueron excluidas porque les faltaba una estructura mínima y porque la disponibilidad de datos financieros suele ser insuficiente para llevar a cabo este tipo de análisis. La población inicial, 44.637 empresas, se obtuvo de la base de datos SABI. De ésta se excluyeron las microempresas, anteriormente comentadas, y las empresas inactivas. De esta forma, la población se redujo a 3.263 empresas. A partir de ahí, se obtuvo una muestra aleatoria representativa de 517 empresas, cuyos directivos respondieron al cuestionario planteado mediante encuesta telefónica.

Las respuestas que interesan a efectos de este trabajo se refieren a cuales son los factores constituye competencia básica y el grado o nivel de intensidad en la gestión llevada a cabo sobre la misma. Para ello, en primer lugar se les cuestionaba a los directivos, ante un listado de recursos y capacidades de carácter tangible e intangible, cuál/es eran su fuente básica de competitividad. Entre aquellas empresas que la asocian a una de las tipologías de capital intelectual, se les pide que evalúen los esfuerzos realizados para fortalecer este factor, planteándose tres niveles de menor a mayor.

Posteriormente, se extrajeron los datos económico-financieros de las empresas de la base de datos SABI. Se recogieron datos de los cinco años siguientes al momento de realización de la encuesta, es decir el periodo 2008-2012. Debido a la falta información para ciertas empresas encuestadas, así como a la desaparición de alguna de ellas, la muestra se ha visto reducida, quedando un total de 344 empresas.

Las variables empleada como indicativa del resultado es el ROA. Se han eliminado los valores extremos, habiéndose considerado como tal, aquellos que se alejan del valor medio en más o menos cinco desviaciones. 
Asimismo se han empleado como variables de control, el tamaño, el sector y la tipología de capital considerada competencia básica. En relación al tamaño se ha considerado una variable dicotomía distinguiendo las PYMEs de las grandes empresas. En cuanto al sector se ha diferenciado, atendiendo al código CNAE, entre el sector primario, el industrial, la construcción y el sector servicios. Finalmente, las tipologías de capital intelectual recogidas son el capital humano, estructural y relacional.

En la Tabla 1 se recoge la ficha técnica del estudio realizado.

Tabla 1

Ficha técnica del estudio

\begin{tabular}{|l|l|}
\hline Población & 3.263 empresas en las empresas del País Vasco \\
\hline Muestra & 517 cuestionarios válidos \\
\hline Técnica de recogida de datos & Encuesta telefónica \\
\hline Calendario & 20 Noviembre 2007 a 14 Enero 2008 \\
\hline Fuente de datos económico-financiero & Base de Datos SABI \\
\hline Calendario & $2008-2012$ \\
\hline Final simple & 344 \\
\hline Error muestral & $4.9 \%$, para un nivel de confianza del $95 \%$ \\
\hline
\end{tabular}

Fuente: Elaboración propia.

\section{RESULTADOS}

Al objeto de analizar la relación entre aquellas empresas que gestionan de un modo más activo su competencia básica y el resultado empresarial, en primer lugar se ha llevado a cabo un análisis descriptivo, recogido en la Tabla 2.

Tabla 2

Gestión de las competencias básicas y el resultado empresarial: Análisis descriptivo

\begin{tabular}{|l|c|c|c|}
\hline & $\mathbf{R O A}_{\mathbf{2 0 0 8 - 1 2}}$ & $\mathbf{R O A}_{\mathbf{2 0 0 8 - 1 0}}$ & $\mathbf{R O A}_{\mathbf{2 0 1 1 - 1 2}}$ \\
\hline $\begin{array}{l}\text { Ha hecho esfuerzos sistemáticos por } \\
\text { fortalecer su competencia básica }\end{array}$ & $2,876 \%$ & $3,950 \%$ & $1,162 \%$ \\
\hline $\begin{array}{l}\text { Ha hecho esfuerzos esporádicos por } \\
\text { fortalecer su competencia básica }\end{array}$ & $1,648 \%$ & $3,631 \%$ & $-0,878 \%$ \\
\hline $\begin{array}{l}\text { No ha hecho esfuerzos por fortalecer } \\
\text { su competencia básica }\end{array}$ & $-0,176 \%$ & $0,792 \%$ & $-0,714 \%$ \\
\hline Total & $2,2295 \%$ & $3,591 \%$ & $0,370 \%$ \\
\hline
\end{tabular}

Fuente: Elaboración propia.

Se observa que el ROA obtenido por las empresas que gestionan más activamente sus competencias básicas es superior, aumentando la diferencia de un modo significativo a partir del segundo año en relación con las empresas que han hecho esfuerzos esporádicos por fortalecerlos, las cuales puntualmente sí 
obtienen buenos resultados pero en un horizonte temporal más amplio, los resultados se asimilan a los de las empresas que no han hecho esfuerzos.

A continuación por mínimos cuadrados ordinarios hemos analizado si existía una relación entre la tipología de gestión llevada a cabo sobre las competencias básicas y la rentabilidad en los tres horizontes temporales considerados. Tras comprobar que no existe multicolinealidad (test de Durbin-Watson), podemos afirmar que las tres variables utilizadas como variables de control no resultan significativas, y que por tanto la única variable que influye en la rentabilidad obtenida es la intensidad de la gestión llevada a cabo sobre las competencias básicas (Tabla 3 ).

Tabla 3

Gestión de las competencias básicas y el resultado empresarial: Análisis descriptivo

\begin{tabular}{|l|c|c|c|}
\hline \multirow{2}{*}{ Variable dependiente } & $\mathbf{R O A}_{\mathbf{2 0 0 8 - 1 2}}$ & $\mathbf{R O A}_{\mathbf{2 0 0 8}-10}$ & $\mathbf{R O A}_{\mathbf{2 0 1 1}-12}$ \\
\cline { 2 - 4 } & $\boldsymbol{\beta}$ no estandarizados & $\boldsymbol{\beta}$ no estandarizados & $\boldsymbol{\beta}$ no estandarizados \\
\hline \multirow{2}{*}{ Constante } &,- 008 &, 025 &,- 051 \\
& $(-0,265)$ & $(0,861)$ & $, 0,203)$ \\
\hline Tipología de Competencias &,- 004 &,- 008 & $(004$ \\
Básica & $(-0,694)$ & $(-1,358)$ &,- 001 \\
\hline Sector &, 001 &, 004 & $(0,830)$ \\
\hline Tamaño & $(-0,251)$ & $(0,719)$ &, 015 \\
&,- 001 & $(-, 012$ & $(0,243)$ \\
\hline Gestión Activa & $(-0,149)$ & $, 01,238)$ &, 011 \\
\hline
\end{tabular}

* significativa al $10 \%$.

** significativa al $5 \%$.

Fuente: Elaboración propia.

\section{DISCUSIÓN DE RESULTADOS Y CONCLUSIONES}

Como argumenta el enfoque de Recursos y Capacidades, los intangibles se han convertido en los recursos básicos para generar una ventaja competitiva. Siguiendo esta corriente, las acciones destinadas a mejorar el modo en que los intangibles son gestionados contribuyen a mejorar los resultados. Es destacable que la mejora no se limita a un ejercicio concreto, sino que las mejoras persisten a lo largo de un horizonte temporal mayor, cinco años, obteniéndose mejoras tanto en los primeros años como posteriormente. Por tanto, la gestión de las competencias básicas asociadas a los intangibles de una empresa debe enmarcarse dentro de un planteamiento estratégico, y el fortalecimiento de las competencias básicas debe integrarse en el plan estratégico.

Consideramos que estas conclusiones son particularmente relevantes ya que a pesar de ser numerosos los trabajos que analizan el stock de intangibles en diferentes tipos de empresas, la implantación de modelos de gestión de intangi- 
bles en la mayoría de los casos se ha limitado a un colectivo limitado de empresas, grandes empresas pertenecientes a sectores intensivos en conocimiento. Sin embargo, los resultados obtenidos que los beneficios de una adecuada gestión no se limitan a este colectivo, por lo que se ha de potenciar la implantación de este tipo de prácticas en todo tipo de empresas.

Por otro lado, cabe señalar que los resultados obtenidos corresponden a un periodo de crisis profundo, lo cual pone de manifiesto que en periodos de mayor dificultad los recursos y capacidades intangibles y su gestión se muestran especialmente significativos.

Este estudio tiene varias limitaciones: en primer lugar, la información sobre la intensidad de la gestión llevada a cabo no se ha cuantificado. Entendemos que la cuantificación no resulta sencilla, ya que más allá de inversiones monetarias, se habrán llevado a cabo inversiones en tiempo, siendo difíciles de cuantificar e integrar éstas inversiones con las anteriores. Entendemos que debemos avanzar en esta línea, y tratar de recoger más evidencias sobre la intensidad de la gestión llevada a cabo. En segundo lugar, debido a que se ha tomado un horizonte temporal amplio, una parte de la muestra ha desaparecido por razones diversas, por lo que únicamente se han podido analizar las empresas que han superado la crisis.

\section{REFERENCIAS BIBLIOGRÁFICAS}

ADAMS, M., MARTIN, H. (2009). "IC: Ready to cross the chasm?". Ponencia presentada en European Conference on Intellectual Capital, INHolland University of Applied Sciences, Haarlem, Holanda, 28-29 Abril.

AMIT, R., SCHOEMAKER, P. (1993). "Strategic assets and organizational rent". Strategic Management Journal, 14, 33-46.

AN, Y., DAVEY, H., EGGLETON, I. R. (2011). "The Effects of Industry Type, Company Size and Performance on Chinese Companies' IC Disclosure: A Research Note". Australasian Accounting Business \& Finance Journal, 5(3), 107-116.

ANDRIESSEN, D., STAM, C. D. (2005). "Intellectual capital of the European Union". Ponencia presentada en McMaster World Congress on the Management of Intellectual Capital and Innovation, Hamilton, Ontario, Canada, 19-21 Enero.

BARNEY, J. B. (1986). "Strategic factor markets: expectations, luck and business strategy". Management Science, 32(10), 1231-1241.

BLAIR, M. M., WALLMAN, S.M. (2001). Unseen Wealth: Report of the Brookings Task Force on Intangibles. Brookings Institute, Washington, DC.

BONTIS, N. (1996). "There's a price on your head: Managing intellectual capital strategically", Business Quarterly, 60(4), 41-47.

BONTIS, N. (1998). "Intellectual capital: An exploratory study that develops measures and models". Management Decision, 36(2), 63-76. 
BONTIS, N.; KEOW W. CH. CH., RICHARDSON, S. (2000). "Intellectual capital and business performance in Malaysian industries". Journal of Intellectual Capital, 1(1), 85-100.

BOSE, S., THOMAS, K. (2007). "Valuation of intellectual capital in knowledge-based firms". Management Decision, 45(9), 1484-1496.

BROOKING, A. (1996). Intellectual capital. Core asset for the third millennium enterprise, International Thomson Business Press, London, UK.

BURTON, K., O'CONNOR, A., ROOS, G. (2013). "An empirical analysis of the IC Navigator approach in practice-a case study of five manufacturing firms". Knowledge Management Research \& Practice, 11(2), 162-174.

CABRITA, M. D. R., BONTIS, N. (2008). "Intellectual capital and business performance in the Portuguese banking industry". International Journal of Technology Management, 43(1), 212-237.

CASTILLA, F., RUIZ, C. (2014). "Una revisión histórico-descriptiva de las empresas pioneras en el tratamiento de intangibles". Intangible Capital, 10(1), 125-154.

CEPEDA, G. (2006) "Competitive Advantage of Knowledge Management", Encyclopedia of Knowledge Management, Vol. 1, No.1, pp. 34-43.

CHATZKEL, J. (2001). "A conversation with Sharon L. Oriel of the Dow Chemical company". Journal of Intellectual Capital, 2(1), 42-52.

COHEN, S., KAIMENAKIS, N. (2007). "Intellectual capital and corporate performance in knowledge-intensive SMEs". The Learning Organization, 14(3), 241-262.

CORRADO, C., HULTEN, C., SICHEL, D. (2009). "Intangible capital and US economic growth". Review of Income and Wealth, 55(3), 661-685.

EDVINSSON, L., MALONE, M. S. (1997). Intellectual capital: Realizing your company's true value by finding its hidden brainpower. HarperCollins Publishers, New York, NY.

FAZLAGIC, A. (2005). "Measuring the intellectual capital of a university". Ponencia presentada en Trends in the Management of Human Resources in Higher Education, París, Francia, 25-26 Agosto.

FIRER, S., WILLIAMS, S. M. (2003). "Intellectual capital and traditional measures of corporate performance". Journal of Intellectual Capital, 4(3), pp. 348-360.

GARCÍA-MECA, E., PARRA, I., LARRÁN, M., MARTÍNEZ I. (2005). 'The explanatory factors of intellectual capital disclosure to financial analysts". European Accounting Review, 14(1), 63-94.

GARCÍA-MERINO, D., RODRÍGUEZ, A., ARREGUI, G., VALLEJO, B. (2008). "Importancia y valoración de los intangibles: la percepción de los directivos". Estudios de Economía Aplicada, 26(3), 27-55.

GARCIA-PARRA, M., SIMO, P., MUNDET, J., AND GUZMAN, J. (2004). "Intangibles: Assets and liabilities". Intangible Capital, 1(3), 70-86.

GRANT, R. M. (1991). "The resource-based theory of competitive advantages: Implications for strategy formulation". California Management Review, 33(3), 114-135.

HANDY, C. (1994). The Empty Raincoat. Hutchinson, London.

HOLSAPPLE, C. W., SINGH, M. (2001). "The knowledge chain model: activities for competitiveness". Expert systems with applications, 20(1), 77-98.

HOLSAPPLE, C., JONES, K., SINGH, M. (2007). "Linking knowledge to competitiveness: knowledge chain evidence and extensions". Holsapple, C. Jones, K., Singh, M. (eds.) Knowledge management in modern organizations. IGP, London, 51-76. 
HOOPES, D. G., Madsen, T. L., Walker, G. (2003) "Guest editors' introduction to the special issue: Why is there a resource-based view? Toward a theory of a competitive advantage", Strategic Management Journal, Vol. 24, (special issue), 889-902.

HUDSON, W. (1993): Intellectual capital. How to build it, enhance it, use it, John Wiley \& Sons, New York, NY.

JARDÓN, C. M., MARTOS, M. S. (2008). "Capital intelectual y resultados empresariales en la cadena de la madera de Oberá (Argentina)". Estudios De Economia Aplicada, 26(3), 141-164.

JONES, N., MEADOW, C., \& SICILIA, M. A. (2009). Measuring intellectual capital in higher education. Journal of Information \& Knowledge Management, 8(02), 113-136.

JOSHI, M., CAHILL, D., SIDHU, J., KANSAL, M. (2013). "Intellectual capital and financial performance: an evaluation of the Australian financial sector". Journal of Intellectual Capital, 14(2), 264-285.

KIANTO, A., ANDREEVA, T., PAVLOV, Y. (2013). "The impact of intellectual capital management on company competitiveness and financial performance". Knowledge Management Research \& Practice, 11(2), 112-122.

KOENIG, M. (2000). "The resurgence of intellectual capital", Information Today, 17(8), disponible en http://www.infotoday.com/it/sep00/koenig.htm

KUJANSIVU, P. (2008). "Operationalising intellectual capital management: choosing a suitable approach", Measuring Business Excellence, 12(2), 25-37.

LEV, B. (2001). Intangibles: Management, measurement and reporting. Brooking Institution Press, Washington D.C.

LEV, B., ZAMBON, S. (2003). "Intangibles and intellectual: capital an introduction to a special issue", European Accounting Review, 12(4), 597-603.

LI, J., PIKE, R., HANIFFA, R. (2008). "Intellectual capital disclosure and corporate governance structure in UK firms". Accounting and Business Research, 38(2), 137159

LÓPEZ, V. R. Y NEVADO, D. (2008). "Análisis integral como sistema de medición y gestión de capitales intangibles en organizaciones y territorios." Estudios de Economía Aplicada, 26(2).

MADITINOS, D., CHATZOUDES, D., TSAIRIDIS, C., \& THERIOU, G. (2011). The impact of intellectual capital on firms' market value and financial performance. Journal of intellectual capital, 12(1), 132-151

MAJDALANY G., HENDERSON, J. (2014). "Voluntary Disclosure of Intellectual Assets and Intellectual Liabilities: Impact on Financial Performance in Publicly Listed Firms in the United Arab Emirates". The Electronic Journal of Knowledge Management, 11(4), 325-334.

MANGENA, M., PIKE, R., LI, J. (2010). Intellectual capital disclosure practices and effects on the cost of equity capital: UK evidence, ICAS, Edimburgo, UK.

MARTÍN, G., DELGADO, M., LÓPEZ, P., NAVAS, J. E. (2011). "Towards 'an intellectual capital-based view of the firm': Origins and nature", Journal of Business Ethics, 98(4), 649-662.

MENTION, A. L., BONTIS, N. (2013). "Intellectual capital and performance within the banking sector of Luxembourg and Belgium". Journal of Intellectual Capital, 14(2), 286-309.

PEÑA, I. (2002). "Intellectual capital and business start-up success". Journal of Intellectual Capital, 3(2) 180-198. 
PETERAF, M. A. (1993). "The Cornerstones of Competitive Advantage: A Resource Based View". Strategic Management Journal, 14, 179-191.

PORTER, M.E. (1980). Competitive Strategy. New York: Free Press.

PORTER, M.E. (1985). Competitive advantage: creating and sustaining superior performance. New York: Free Press.

PRAHALAD, C. K. (1983). "Developing strategic capability: an agenda for Management", Human Resources Management, Vol. 22, pp. 237-254.

PRAHALAD, C. K., HAMEL, G. (1994). "Strategy as a field study: Why search for a new paradigm?”. Strategic Management Journal, 15(S2), 5-16.

PRAHALAD, C.K., HAMEL, G. (1990). "The Core Competence of the Corporation". Harvard Business Review, May-June, 79-91.

RAMÍREZ, Y.; SANTOS. J. F. Y TEJADA, Á. (2011). "Beneficios y costes de la divulgación de información sobre capital intelectual: Evidencia empírica en las universidades públicas españolas”. Estudios de Economía Aplicada, 29 (3), 1-28.

RASTOGI, P. N. (2000). Knowledge management and intellectual capital-the new virtuous reality of competitiveness. Human Systems Management, 19(1), 39-48.

REED, K. K., LUBATKIN, M., SRINIVASAN, N. (2006). "Proposing and testing an intellectual capital-based view of the firm". Journal of Management Studies, 43(4), 867-893.

RIAHI-BELKAOUI, A. (2003). "Intellectual capital and firm performance of US multinational firms: a study of the resource-based and stakeholder views". Journal of Intellectual capital, 4(2), 215-226.

ROOS, G.; ROOS, J., EDVINSSON, L., DRAGONETTI, N. C. (1997). Intellectual capital. Navigating in the new business landscape, McMillan, London, UK.

SÁNCHEZ-MEDINA, A. J. (2003). Modelo para la medición del capital intelectual de territorios insulares: una aplicación al caso de Gran Canaria. Doctoral Dissertation, Las Palmas de Gran Canaria: University of Las Palmas de Gran Canaria.

SELEIM, A. A., KHALIL, O. E. (2011). "Understanding the knowledge management-intellectual capital relationship: a two-way analysis". Journal of Intellectual Capital, 12(4), 586-614.

SHARABATI, A. A. A., JAWAD, S. N., BONTIS, N. (2010). Intellectual capital and business performance in the pharmaceutical sector of Jordan. Management Decision, 48(1), 105-131.

SIMARO, J. D., TONELLI, O. E., CARÚS RIBALAYGUA, L. (2012). Gestión de intangibles en pymes turísticas: Un caso de aplicación de las directrices MERITUM. Estudios y perspectivas en turismo, 21(1), 249-269.

SIRMON, D. G., GOVE, S., HITT, M. A. (2008). Resource management in dyadic competitive rivalry: The effects of resource bundling and deployment. Academy of Management Journal, 51(5), 919-935.

SONNIER, B. M., CARSON, K. D., CARSON, P. P. (2007). "Accounting for intellectual capital: The relationship between profitability and disclosure", The Journal of Applied Management and Entrepreneurship, 12(2), 3-14.

STÅHLE, S., STÅHLE, P. (2012). Towards measures of national intellectual capital: an analysis of the CHS model. Journal of Intellectual Capital, 13(2), 164-177.

STEENKAMP, N., KASHYAP, V. (2010). Importance and contribution of intangible assets: SME managers' perceptions. Journal of Intellectual Capital, 11(3), 368-390. 
STEWART, R. A. Y MOHAMED, S. (2001): "Utilizing the Balanced Scorecard for IT/IS performance evaluations in construction", Construction Innovation, Vol. 1, N $\mathrm{N}^{\circ} 3$, pp. 147-163.

STEWART, T. A. (1997): Intellectual capital. The new wealth of organizations, Doubleday/Currency, New York, NY.

SULLIVAN, P. H. (1998). Profiting from intellectual capital: Extracting value from innovation. John Wiley \& Sons, New York, NY.

SVEIBY, K. E. (2011). Methods for Measuring Intangible Assets. Disponible http://www.sveiby.com/articles.Intangible-Methods html.

TEJEDO, F. T., ALFARO, E. A. (2014). Asociación entre las características empresariales y la divulgación sobre Capital Intelectual: Un estudio de las empresas del Ibex 35. Estudios de Economía Aplicada, 32(1), 371-398.

TULL, A. A., DUMAY, J. C. (2007). "Does Intellectual Capital management 'make a difference'? A critical case study application of strucuturation theory". The Electronic Journal of Knowledge Management, 5(4), 515-526.

WANG, M. S. (2011). "Intellectual capital and firm performance". Ponencia en Annual Conference on Innovations in Business and Management, Londres, UK, 26-27 Enero.

WANG, W.-Y., CHANG, CH. (2005). "Intellectual capital and performance in causal models: Evidence from the information technology industry in Taiwan", Journal of Intellectual Capital, 6(2), 222-236.

WERNERFELD, A. (1984). "A resource based view of the firm", Strategic Management Journal, Vol. 5, No. 2, pp. 171-180.

WIIG, K. (1997a). "Integrating intellectual capital and knowledge management", Long Range Planning, 30(3), 399-405.

WIIG, K. (1997b). "Knowledge management: an introduction and perspective". Journal of Knowledge Management, 1(1), 6-14

WILLIAMS, S. M. (2001). Is intellectual capital performance and disclosure practices related?" Journal of Intellectual capital, 2(3), 192-203. 
